\title{
Helping researchers to solve their structures: automation and user guidance in CCP4 Cloud
}

\author{
O. Kovalevskiy, A. Lebedev, E. Krissinel \\ CCP4, Rutherford Appleton Laboratory, UKRI STFC, Harwell Campus, Didcot, Oxfordshire, OX11 0QX UK \\ eugene.krissinel@stfc.ac.uk
}

Structure solution in macromolecular crystallography is not always a straightforward process and it may be rather difficult for structural biologists without advanced training. A trained crystallographer exploits an extended set of approaches and tricks, based on the analysis of several indicators, general assessment of the case, and developed strategies for dealing with a particular class of problems. If such an approach, used by an expert, can be formalised in terms of an algorithm, then it can be implemented as a computer program or automated user advice system to help users solving structures quicker and with a higher success rate. It is not surprising then, that programs for macromolecular crystallography are moving towards full automation, taking off burden from researchers and lowering the entry barriers for novice users.

During the last few years, substantial progress has been made towards automation of the whole macromolecular structure determination process. There are a number of examples of successful automatic solutions for various stages of structure determination, such as molecular replacement, experimental phasing, and refinement [1-5].

In this communication, we report two novel automation features implemented in CCP4 Cloud [6], the new system for solving macromolecular structures online, released with CCP4 Software Suite 7.1 in 2020. Automated user advice framework, named Verdicts, provides simple graphical representation of results quality with detailed analysis of points for improvement as part of every task (e.g., refinement) report. The analysis includes suggestions on what could be done in order to improve the result (i.e., which parameters could be optimised). Then, the task can be re-run with the suggested parameters, which can be further adjusted by the user as appropriate.

Another automation feature, Workflows, was designed for unfolding structure solution Projects, or their parts, automatically using user-supplied data. Such automatically initiated and unfolded Projects may include a number of tasks, arranged in branching Project Trees as if this were done by the user themselves. In common cases without complications, this may result in structure solved, and if not, then a starting Project is offered to the user for analysis and further manipulations, where simple, first-order structure solution attempts are already performed. Any task or branch of the starting Project may be cloned and re-run with optimized parameters, and new tasks may be added as needed. Workflows combine automation and human expert skills, and, therefore, represent an excellent starting point for users with different level of expertise, ranging from novices to experienced crystallographers. Workflows are particularly useful in a common case of processing large sets of isomorphous crystals, because, once structure is solved in one crystal, the process is well-repeatable in systems with moderate modifications.

[1] Winter, G., Lobley, C. M. \& Prince, S. M. (2013). Acta Crystallogr D Biol Crystallogr 69, 1260.

[2] Long, F., Vagin, A. A., Young, P. \& Murshudov, G. N. (2008). Acta Crystallogr D Biol Crystallogr 64, 125.

[3] Keegan, R. M., Long, F., Fazio, V. J., Winn, M. D., Murshudov, G. N. \& Vagin, A. A. (2011). Acta Crystallogr D Biol Crystallogr 67, 313.

[4] Minor, W., Cymborowski, M., Otwinowski, Z. \& Chruszcz, M. (2006). Acta Crystallogr D Biol Crystallogr 62, 859.

[5] Wojdyr, M., Keegan, R., Winter, G., Ashton, A., Lebedev, A. \& Krissinel, E. (2014). Acta Crystallographica Section A 70, C1447.

[6] Krissinel, E., Uski, V., Lebedev, A., Winn, M. \& Ballard, C. (2018). Acta Crystallographica Section D 74, 143.

Keywords: crystallographic computing; distributed computing; automation; structure determination.

CCP4 Cloud was developed with funding from BBSRC UK (Grant BB/S007040/1) and CCP4. 